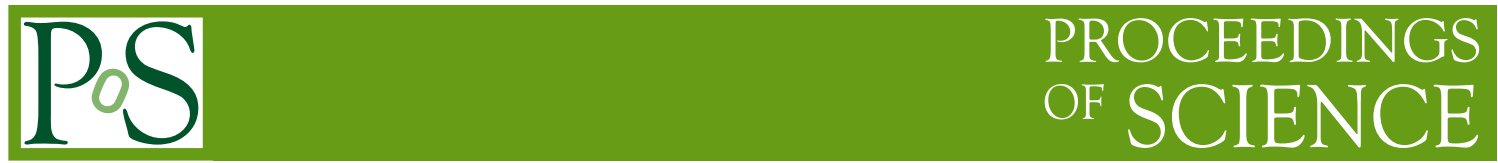

\title{
Bethe Ansatz and Scattering in Stringy Sigma-Models
}

\section{K. Zarembo*t}

Department of Theoretical Physics, Uppsala University

Box 803, SE-751 08 Uppsala, Sweden

E-mail: Konstantin.Zarembo@teorfys.uu.se

Two simple reductions of string theory in $A d S_{5} \times S^{5}$ are reviewed, with emphasis on the scattering, pseudovacuum, Bethe ansatz, and finite-size effects.

Bethe Ansatz: 75 years later

October 19-21 2006,

Brussels, Belgium

\footnotetext{
* Speaker.

${ }^{\dagger}$ Also at ITEP, Moscow, 117259 Bol. Cheremushkinskaya 25, Moscow, Russia
} 


\section{Introduction}

The Bethe ansatz [1] is a general tool to solve $(1+1)$-dimensional integrable field theories [2-4]. Its use in the AdS/CFT duality might appear quite unexpected, since both sides of the duality are not two-dimensional: the AdS/CFT correspondence relates supersymmetric Yang-Mills theory in four dimensions to string theory in ten dimensions (the string background is the direct product $A d S_{5} \times S^{5}$ with the constant Ramond-Ramond (RR) flux) [5]. However, first-quantized, non-interacting strings are described by a two-dimensional field theory on their world-sheet, and in the case of the $A d S_{5} \times S^{5}$ background this theory [6] is an integrable sigma-model [7]. Integrability in the SYM theory is more intricate and arises as a subsidiary symmetry in the mixing problem for gauge-invariant local operators, which can be reformulated in terms of a one-dimensional spin chain in the large- $N$ limit $[8,9]$.

There is one feature of the AdS/CFT duality that clearly distinguishes it from other, more traditional uses of the Bethe ansatz. Usually one is mostly interested in the bulk, macroscopic quantities, such as the spectrum of excitations, thermodynamics, etc. which determine the behavior of the system in an infinite volume. This is not sufficient in the AdS/CFT correspondence. In order to quantize closed strings we need to impose periodic boundary conditions on the world-sheet coordinates. The states of the spin chain parameterize single-trace composite operators and are also naturally periodic.

It is not surprising the infinite-volume limit in the AdS/CFT context is much better understood. Interactions are then characterized by the S-matrix, which is highly constraint by symmetries and in integrable system in addition factorizes into two-particle blocks [10]. The non-perturbative S-matrix of the AdS/CFT correspondence [11] is known [12] and was found by combining symmetries, dynamical information and a certain amount guess-work. The S-matrix determines the common spectrum of $\mathscr{N}=4 \mathrm{SYM}$ and strings in $A d S_{5} \times S^{5}$ via Bethe ansatz equations [13], which are exponentially accurate for sufficiently long strings/gauge operators $[14,15]$. These notes are based on [16] and are devoted to two simpler sigma-models (that have arisen in the AdS/CFT duality) in which scattering theory completely determines the exact spectrum even if the volume is finite.

In order to estimate the accuracy of the infinite-volume limit, let me start with the simplest possible example: one-dimensional quantum mechanics with the potential

$$
V(x)=\sum_{s=-\infty}^{+\infty} U(x+s L)
$$

where $U(x)$ short-ranged and exponentially decays at infinity. For simplicity I will consider the semiclassical limit (the conclusion does not change if quantum effects are taken into account exactly). When periodic boundary conditions, written conveniently as $\Psi(L / 2) / \Psi(-L / 2)=1$, are imposed on the semiclassical wave-function

$$
\Psi(x) \sim \exp \left(\frac{i}{\hbar} \int_{-L / 2}^{x} d x^{\prime} \sqrt{p^{2}-2 m V\left(x^{\prime}\right)}\right),
$$

the momentum gets quantized according to the Bohr-Sommerfeld rule:

$$
\mathrm{e}^{i \Phi_{L}(p)}=1, \quad \Phi_{L}(p)=\frac{1}{\hbar} \int_{-L / 2}^{L / 2} d x \sqrt{p^{2}-2 m V(x)} .
$$


The quantization of momentum, together with the dispersion relation, determines admissible energy levels of the periodic system: $\varepsilon\left(p_{n}\right)=p_{n}^{2} / 2 m$, where $\left\{p_{n}\right\}$ are solutions to (1.2).

The summation that makes the potential periodic stands in (1.2) under the square root. Taking the summation out and using

$$
\sum_{s=-\infty}^{+\infty} \int_{-L / 2}^{L / 2} d x f(x+s L)=\int_{-\infty}^{+\infty} d x f(x)
$$

yields an asymptotic approximation which relates the phase of the wave function in the potential $V$ :

$$
\Phi_{L}(p) \approx p L+\Delta(p),
$$

to the scattering phase shift for the potential $U$ on an infinite line:

$$
\Delta(p)=\frac{1}{\hbar} \int_{-\infty}^{+\infty} d x\left(\sqrt{p^{2}-2 m U(x)}-p\right)
$$

The momentum quantization condition in that approximation is

$$
\mathrm{e}^{i p L}=\mathrm{e}^{-i \Delta(p)}
$$

Its multiparticle generalization is the set of Bethe equations

$$
\mathrm{e}^{i p_{j}}=\prod_{k \neq j} \mathrm{e}^{-i \Delta\left(p_{j}, p_{k}\right)}, \quad E=\sum_{j} \varepsilon\left(p_{j}\right)
$$

where $\Delta\left(p_{j}, p_{k}\right)$ is the scattering phase shift of the $j$ th particle on the $k$ th particle and $\varepsilon=\varepsilon(p)$ is the one-particle dispersion relation. This equation holds under an additional assumption that the multiparticle scattering factorizes and reduces to a sequence of pairwise interactions, which is always the case for integrable systems [10].

The accuracy of the asymptotic approximation can be estimated by comparing (1.2) to (1.3) and (1.4):

$$
\Phi_{L}(p)-p L-\Delta(p) \approx \frac{m}{\hbar} \int_{-L / 2}^{L / 2} d x \sum_{s \neq 0} U(x+s L)\left(\frac{1}{p}-\frac{1}{\sqrt{p^{2}-2 m U(x)}}\right) \sim \mathrm{e}^{-L / R},
$$

where $R$ is the range of the potential. Thus, if the potential is short-ranged, the corrections are exponential. The cricial point for our further analysis is that in one distinguished case (1.5), (1.6) are exact: namely, when interactions are completely localized (the potential is the delta function), (1.7) identically turns to zero.

In quantum field theory with local interactions the potential is the delta function at tree level, but vacuum polarization smears interactions over a finite range, which is determined by the mass of virtual particles ${ }^{1}: R=1 / 2 \mathrm{~m}$. A way out is to define a reference state (the pseudovacuum) in which

\footnotetext{
${ }^{1}$ Typically, the potential is generated by pair creation. One could conclude from the discussion above that the accuracy of the asymptotic Bethe ansatz is then $\mathrm{e}^{-2 m L}$, but virtual pairs that travel around the compact direction produce even stronger effect in the dispersion relation of a single particle and lead to finite-size corrections that scale as $\mathrm{e}^{-m L}$, see [14] for the discussion of this effect in the context of the AdS/CFT correspondence.
} 
scattering is simple and the vacuum is not polarized ${ }^{2}$. An example of pseudovacuum is the empty Fermi sea. Since draining the Fermi sea removes all anti-particles, there is no vacuum polarization. Of course, an empty Ferminsea is a highly unphysical and unstable state. In order to reconstruct the physical spectrum, the Fermi sea has to be refilled with particles so that no negative-energy states are left, but this can be done with the help of the Bethe equations which in the pseudovacuum are exact even in the finite volume. Many integrable field theories in two-dimensions can be solved this way (Korepin's solution of the massive Thirring model [17, 18] is a typical example of this sort) $[3,4]$. It is currently not known how to construct the pseudovacuum in string theory on $A d S_{5} \times S^{5}$. Hopefully finding such state will help in understanding the finite-size spectrum of the AdS/CFT correspondence as well.

I shall describe how to define the pseudovacuum and to compute the bare S-matrix for two sigma models that arise as truncations of string theory in $A d S_{5} \times S^{5}$. The first is the non-relativistic Landau-Lifshitz (LL) model, and the second is the string sigma-model on $S^{3}$, of which the LL models is a non-relativistic limit.

\section{Landau-Lifshitz model}

The macroscopic spin waves with large wave number are described in one dimension by the LL equation [19]:

$$
\partial_{t} n_{i}=\varepsilon_{i j k} n_{j} \partial_{x}^{2} n_{k}, \quad \mathbf{n}^{2}=1
$$

The LL model is the low-energy effective theory of the Heisenberg Hamiltonian, which arises in the AdS/CFT correspondence as the one-loop mixing matrix in the $s u(2)$ sector of $\mathscr{N}=4 \mathrm{SYM}$ [8]. On the string side of the AdS/CFT correspondence, the LL equation describes fast-moving strings on the $R^{1} \times S^{3}$ subspace of $A d S_{5} \times S^{5}$ [20] (see also the next section).

The equation (2.1) is classically integrable, but I would like to treat the LL model as a quantum field theory ${ }^{3}$. The LL equations are first order in time derivatives, which means that $n_{i}$ are phasespace variables. To formulate the path integral for the LL model one needs to introduce the WessZumino-type functional. Let me shortly review this construction, following the book [22].

The Wess-Zumino term (in this context) is defined through a non-local functional

$$
C_{q}(\mathbf{n})=-\frac{1}{2} \int_{0}^{1} d \xi \varepsilon_{i j k} n_{i} \partial \xi n_{j} \partial_{q} n_{k}
$$

For periodic boundary conditions in time direction, the WZ term can be written as a two-dimensional integral such that the time direction is the boundary of the integration region:

$$
\int d t C_{t}(\mathbf{n})=-\frac{1}{4} \int_{D} \varepsilon_{i j k} n_{i} d n_{j} \wedge d n_{k} .
$$

Since the integrand is a closed form, a local variation of $\mathbf{n}$ inside $D$ does not change the WZ functional (global variations can change the WZ action by integer multiples of $2 \pi$ ). The manifestly

\footnotetext{
${ }^{2}$ This is a rather loose definition. A precise notion of the pseudovacuum can be only given in the algebraic Bethe ansatz framework [2].

${ }^{3}$ The LL field theory is a non-relativisitc non-linear sigma-model with the $S^{2}$ target space. The quantum LL model on the hyperbolic space was solved by the algebraic Bethe ansatz in [21].
} 
local form of the $\mathrm{WZ}$ action can be obtained at the price of losing manifest $S O(3)$ invariance. If one solves the constraint $\mathbf{n}^{2}=1$ for $n_{3}=\sqrt{n_{1}^{2}+n_{2}^{2}}$, the integrand can be written as a total derivative

$$
\frac{1}{2} \varepsilon_{i j k} n_{i} d n_{j} \wedge d n_{k}=\frac{d n_{1} \wedge d n_{2}}{n_{3}}=d\left(\frac{n_{1} d n_{2}-n_{2} d n_{1}}{1+n_{3}}\right) .
$$

and thus the auxiliary integration in the WZ functional can be eliminated:

$$
\int d t C_{t}(\mathbf{n})=\frac{1}{2} \int d t \frac{n_{2} \partial_{t} n_{1}-n_{1} \partial_{t} n_{2}}{1+n_{3}} .
$$

The action of the LL model is

$$
S=\int d^{2} x\left[C_{t}(\mathbf{n})-\frac{1}{4}\left(\partial_{x} \mathbf{n}\right)^{2}\right]
$$

Variation of this action yields the equations of motion (2.1). We would like to consider it as an action for a non-linear quantum field theory in $1+1$ dimensions. The change of variables

$$
\varphi=\frac{n_{1}+i n_{2}}{\sqrt{2+2 n_{3}}} \quad, \quad n_{3}=1-2|\varphi|^{2},
$$

gets rid of the non-linearities in the kinetic term [23]. Performing it in (2.5), (2.6), we find:

$S=\int d^{2} x\left\{\frac{i}{2}\left(\varphi^{*} \partial_{t} \varphi-\partial_{t} \varphi^{*} \varphi\right)-\left|\partial_{x} \varphi\right|^{2}-\frac{1}{4} \frac{2-|\varphi|^{2}}{1-|\varphi|^{2}}\left[\left(\varphi^{*} \partial_{x} \varphi\right)^{2}+\left(\partial_{x} \varphi^{*} \varphi\right)^{2}\right]-\frac{1}{2} \frac{|\varphi|^{4}\left|\partial_{x} \varphi\right|^{2}}{1-|\varphi|^{2}}\right\}$.

This is the action of an interacting field theory of a single scalar field. Its non-relativistic character leads to some important non-renormalization properties.

The field theory at hand describes a single non-relativistic particle with $\varepsilon=p^{2}$, and we can build perturbation theory on top of the empty state that contains no particles and is annihilated by the field operator:

$$
\varphi|0\rangle=0
$$

The Feynman propagator will be purely retarded and will have only one pole in the momentum representation:

$$
D(\omega, p)=\frac{i}{\omega-p^{2}+i \varepsilon} .
$$

This leaves no room for vacuum polarization (the vacuum is completely empty): any diagram that contains a closed loop of $\varphi$ vanishes (fig. 1), because poles of all propagators lie on one side of the integration contour over $\omega$ and by contour argument the integral yields zero [19].

The above argument has three important consequences $[19,16]$ :

- The ground state energy is not renormalized: $E_{\mathrm{vac}}=0$. This is consistent with the fact that the ferromagnetic vacuum is the exact zero-energy eigenstate of the Heisenberg Hamiltonian.

- The one particle Green's function is not renormalized. Hence, the dispersion relation $\varepsilon=p^{2}$ does not receive quantum corrections.

- The two-body S-matrix is given by the sum of bubble diagrams in fig. 2 . 


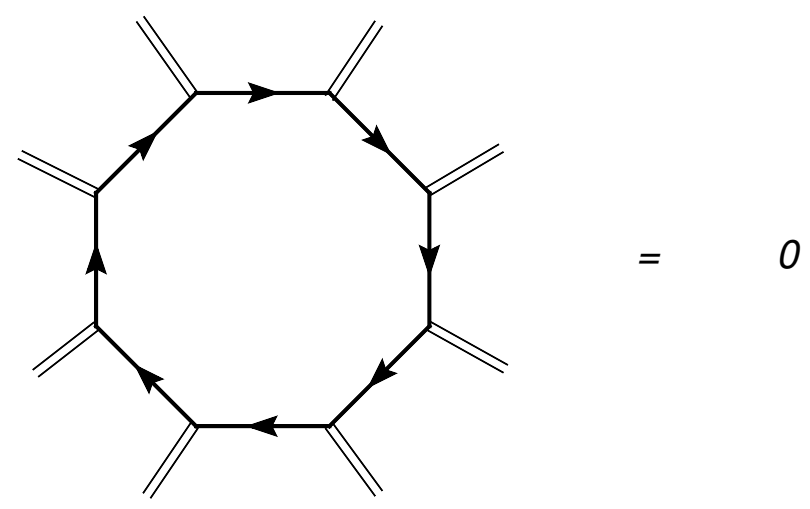

Figure 1: Vacuum polarization is absent in the LL model.

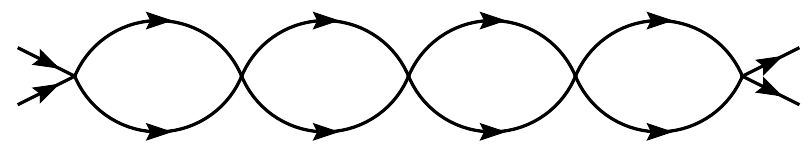

Figure 2: The $2 \rightarrow 2$ S-matrix is a sum of bubble diagrams.

These properties are almost obvious. A formal proof can be given by cutting a generic diagram and counting intermediate propagators.

To compute the S-matrix we may truncate the non-polynomial action (2.8) at the fourth order in the fields:

$$
\mathscr{L}=\frac{i}{2}\left(\varphi^{*} \partial_{t} \varphi-\partial_{t} \varphi^{*} \varphi\right)-\left|\partial_{x} \varphi\right|^{2}-\frac{1}{2}\left[\left(\varphi^{*} \partial_{x} \varphi\right)^{2}+\left(\partial_{x} \varphi^{*} \varphi\right)^{2}\right]+\mathscr{O}\left(\varphi^{6}\right),
$$

since only the quartic vertices contribute to the chain of bubbles. The essentially non-linear nature of the LL model turns out to be unimportant! The S-matrix can be calculated by summing the chain of bubble diagrams as done in [16] (this calculation is very similar to the computation of the S-matrix in the non-linear Schrödinger model [24] of Thirring model in [18]). The derivatives in the vertices somewhat complicate the calculation, but there is a simple argument that allows to compute the exact S-matrix in essentially no time. The argument is based on unitarity, fig. 3, and the fact the sum of bubbles is a geometric series. The unitarity relates the bubble to the tree-level scattering amplitude

$$
\begin{aligned}
& 2 i p p^{\prime}(2 \pi)^{2} \delta^{(2)}\left(p^{\mu}+p^{\prime \mu}-k^{\mu}-k^{\prime \mu}\right) \\
& =\frac{2 i p p^{\prime}}{\frac{\partial \varepsilon(p)}{\partial p}-\frac{\partial \varepsilon\left(p^{\prime}\right)}{\partial p^{\prime}}}(2 \pi)^{2}\left(\delta(p-k) \delta\left(p^{\prime}-k^{\prime}\right)+\delta\left(p-k^{\prime}\right) \delta\left(p^{\prime}-k\right)\right),
\end{aligned}
$$

which gives for the tree-level T-matrix:

$$
T=\frac{2 p p^{\prime}}{p-p^{\prime}}
$$

and since the sum of bubbles is a geometric series, we find the exact S-matrix:

$$
S=\frac{1+\frac{i T}{2}}{1-\frac{i T}{2}}=\frac{\frac{1}{p}-\frac{1}{p^{\prime}}-i}{\frac{1}{p}-\frac{1}{p^{\prime}}+i}
$$




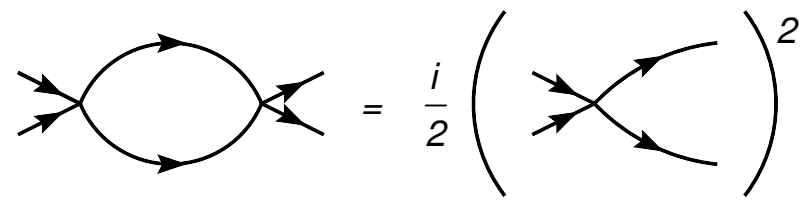

Figure 3: Optical theorem for the bubble diagram.

The Bethe equations resulting from this S-matrix are most conveniently written in terms of the spectral variable $u=1 / p$ :

$$
\mathrm{e}^{i L / u_{j}}=\prod_{k \neq j} \frac{u_{j}-u_{k}+i}{u_{j}-u_{k}-i} .
$$

The energy and the momentum are then given by

$$
E=\sum_{j} \frac{1}{u_{j}^{2}}, \quad P=\sum_{j} \frac{1}{u_{j}} .
$$

It is now easy to show that the reference state (2.9) is a pseudovacuum and not the true ground state of the LL model [16]. Let us consider a bound state of two particles, which is described by a 2-string configuration of Bethe roots:

$$
u_{1,2}=v \pm \frac{i}{2} .
$$

This is an exact solution of the Bethe equations in the strict thermodynamic $(L \rightarrow \infty)$ limit. The energy and the momentum of the 2-string are:

$$
\begin{aligned}
& E_{2-\text { string }}=\frac{1}{\left(v+\frac{i}{2}\right)^{2}}+\frac{1}{\left(v-\frac{i}{2}\right)^{2}}=\frac{2 v^{2}-\frac{1}{2}}{\left(v^{2}+\frac{1}{4}\right)^{2}}, \\
& P_{2-\text { string }}=\frac{1}{v+\frac{i}{2}}+\frac{1}{v-\frac{i}{2}}=\frac{2 v}{v^{2}+\frac{1}{4}} .
\end{aligned}
$$

The momentum of the 2-string is always smaller that 2 and there are two branches of the dispersion relation that describe two kinds of excitations

$$
\varepsilon_{ \pm}(p)=p^{2} \pm 2 \sqrt{4-p^{2}}-4
$$

The two branches arise from $v \gtrless 1 / 2$. The $\varepsilon_{-}$branch has negative energy, so that the true ground state is a Fermi sea of the 2-strings.

\section{Faddeev-Reshetikhin model}

In this section I discuss string theory on $S^{3} \times R$. We should probably explain what do I mean by that because $S^{3} \times R$ is not a string background. There are no problems with classical strings, in fact $S^{3} \times R$ can be regarded as a subspace of $\mathrm{AdS}_{5} \times S^{5}$, but quantization leads to UV divergences and non-zero beta-function. Although the resulting model cannot be interpreted as string theory, it is an interesting example of two-dimensional integrable field theory first considered in [25]. 
The embedding the string world-sheet in the sphere can be parameterized by a group element of $S U(2), g(t, x)$. The time coordinate can be eliminated by imposing the temporal gauge:

$$
X^{0}=\kappa t
$$

The parameter $\kappa$ plays the role of the energy of the string. In terms of the left currents

$$
j_{a}=g^{-1} \partial_{a} g
$$

the equations of motion of the string read

$$
\begin{aligned}
\partial_{a} j^{a} & =0, \\
\partial_{a} j_{b}-\partial_{b} j_{a}+\left[j_{a}, j_{b}\right] & =0 .
\end{aligned}
$$

In addition we should impose the Virasoro constraints:

$$
\operatorname{tr} j_{ \pm}^{2}=-2\left(\partial_{ \pm} X^{0}\right)^{2}=-2 \kappa^{2}
$$

where $\partial_{ \pm}=\partial_{t} \pm \partial_{x}$ are the light-cone derivatives $\left(x^{ \pm}=(t \pm x) / 2\right)$. Accordingly, $j_{ \pm}$are the lightcone components of the $s u(2)$ current. It is this extra condition that distinguishes the FaddeevReshetikhin model from the ordinary principal chiral field. The Virasoro constraints are solved by

$$
j_{ \pm}=i \kappa \mathbf{S}_{ \pm} \cdot \sigma
$$

where $\mathbf{S}_{+}$and $\mathbf{S}_{-}$are three-dimensional vectors of unit norm: $\mathbf{S}_{ \pm}^{2}=1$, which as a consequence of (3.3) satisfy

$$
\partial_{ \pm} S_{\mp}^{i} \pm \kappa \varepsilon^{i j k} S_{-}^{j} S_{+}^{k}=0 .
$$

The equations of motion (3.6) are very similar to the LL equation (2.1), and can be obtained from an action with two light-cone WZ terms [16]:

$$
S=\int d^{2} x\left(C_{+}\left(\mathbf{S}_{-}\right)+C_{-}\left(\mathbf{S}_{+}\right)-\frac{\kappa}{2} \mathbf{S}_{+} \cdot \mathbf{S}_{-}\right),
$$

where $C_{ \pm}$are defined in (2.2). We should stress that this is not the standard action of the sigmamodel on $S^{3}$. It leads to a non-standard Poisson brackets between the components of the $s u(2)$ currents [25]. The Hamiltonian and the momentum derived from this action are also non-standard, but they do arise as the first two commuting charges in the integrable hierarchy associated with the equations (3.3) [25, 26], in contradistinction to the standard energy and momentum of the chiral field which have no good interpretation in terms of integrability and moreover are fixed by the Virasoro constraints. We shall regard (3.7) as a starting point for quantization, which obviously differs from the usual quantization of the sigma-model on $S^{3}$.

To develop perturbation theory for the FR model, we perform the same change of variables as in previous section:

$$
\phi_{ \pm}=\frac{S_{ \pm}^{1}+i S_{ \pm}^{2}}{\sqrt{2+2 S_{ \pm}^{3}}} \quad, \quad S_{ \pm}^{3}=1-2\left|\phi_{ \pm}\right|^{2}
$$


Upon this change of variables, the WZ term becomes a canonically normalized first-order action for two complex scalar fields $\phi_{+}$and $\phi_{-}$:

$$
\begin{aligned}
S= & \int d^{2} x\left[\frac{i}{2}\left(\phi_{+}^{*} \partial_{-} \phi_{+}-\partial_{-} \phi_{+}^{*} \phi_{+}+\phi_{-}^{*} \partial_{+} \phi_{-}-\partial_{+} \phi_{-}^{*} \phi_{-}\right)\right. \\
& -\kappa \sqrt{\left(1-\left|\phi_{+}\right|^{2}\right)\left(1-\left|\phi_{-}\right|^{2}\right)}\left(\phi_{+}^{*} \phi_{-}+\phi_{-}^{*} \phi_{+}\right)+\kappa\left(\left|\phi_{+}\right|^{2}+\left|\phi_{-}\right|^{2}\right) \\
& \left.-2 \kappa\left|\phi_{+}\right|^{2}\left|\phi_{-}\right|^{2}\right] .
\end{aligned}
$$

This action can be cast into a very concise form if we combine $\phi_{+}$and $\phi_{-}$into a two-component commuting spinor:

$$
\phi=\left(\begin{array}{l}
\phi_{-} \\
\phi_{+}
\end{array}\right) .
$$

Then (3.9) becomes a Dirac-like action

$$
S=\int d^{2} x\left(i \bar{\phi} \not \supset \phi-\kappa \bar{\phi} \phi-\frac{\kappa}{2} \bar{\phi} \gamma^{\mu} \phi \bar{\phi} \gamma_{\mu} \phi+O\left(\phi^{6}\right)\right) .
$$

The covariant derivative contains a field-dependent chemical potential:

$$
D_{0}=\partial_{0}-i \kappa-\frac{i \kappa}{2} \bar{\phi} \phi \quad, \quad D_{1}=\partial_{1}
$$

This action describes a single charged particle and its anti-particle with the dispersion relations

$$
\varepsilon=\sqrt{p^{2}+\kappa^{2}}-\kappa \quad(\text { particle })
$$

and

$$
\varepsilon=\sqrt{p^{2}+\kappa^{2}}+\kappa \quad(\text { anti-particle }) .
$$

The mass gap for the particles is offset to zero by the chemical potential. The energy of antiparticles is shifted in the opposite direction, so that anti-particles decouple at low energies and momenta.

The low-energy theory for modes with $\varepsilon \ll \kappa$ is the LL model. This can shown by separating big and small components of the spinor:

$$
\varphi=\frac{1+\gamma^{0}}{2} \phi, \quad \chi=\frac{1-\gamma^{0}}{2} \phi
$$

The Lagrangian then becomes

$$
\mathscr{L}=i \varphi^{*} \partial_{0} \varphi+i \chi^{*} \partial_{0} \chi+i \varphi^{*} \partial_{1} \chi+i \chi^{*} \partial_{1} \varphi+2 \kappa|\chi|^{2}+\frac{\kappa}{2}\left(\varphi^{* 2} \chi^{2}+\chi^{* 2} \varphi-|\chi|^{4}\right) .
$$

Integrating out $\chi$ we arrive at

$$
\mathscr{L}=i \varphi^{*} \partial_{0} \varphi-\frac{1}{2 \kappa}\left|\partial_{1} \varphi\right|^{2}-\frac{1}{8 \kappa}\left[\left(\varphi^{*} \partial_{1} \varphi\right)^{2}+\left(\partial_{1} \varphi^{*} \varphi\right)^{2}\right]+\ldots
$$

which is the same as (2.11) up to rescalings of $\varphi$ and time. 
There is a big difference though between the sigma-model and its non-relativistic limit. The anti-particles that show up in loops in the sigma-model destroy simple non-renormalization properties present in the non-relativistic case. One can prevent this from happening by defining the pseudovacuum, which is annihilated by both components of $\phi$ :

$$
\phi_{ \pm}|0\rangle=0 .
$$

This condition leaves all anti-particle states empty, and consequently some of the excitations on top of this "wrong" vacuum carry negative energy. This is the price to pay for suppressing the vacuum polarization which allows us to compute the S-matrix by summation of the bubble diagrams. We can subsequently find the set up Bethe equations, and then fill the vacuum to eliminate all negativeenergy levels from the spectrum.

The choice of the reference state leads to a the non-relativistic pole prescription for the propagator, in which both poles (particle and would-be anti-particle) are encircled in the same direction. The field-independent part of the chemical potential in (3.12) can then be eliminated by a shift of the integration variable $k^{0}$, after which the dispersion relations become relativistically invariant. But interactions (through the field-dependent part of the chemical potential in (3.12)) still break the Lorentz invariance. To treat positive and negative energy states on the same footing, it is convenient to introduce the rapidity: $p^{0}=\kappa \cosh \theta, p^{1}=\kappa \sinh \theta$. For positive-energy modes $\theta$ is real, while negative energy states correspond to $\operatorname{Im} \theta=\pi$. The scattering wavefunctions for both positive and negative energy states can be taken in the form

$$
u(p)=\sqrt{\kappa}\left(\begin{array}{c}
e^{-\theta / 2} \\
e^{\theta / 2}
\end{array}\right)
$$

Again we can deduce the exact S-matrix from the tree-level scattering amplitude

$$
T=\kappa\left(\frac{\cosh \frac{\theta+\theta^{\prime}}{2}}{\sinh \frac{\theta-\theta^{\prime}}{2}}-\operatorname{coth} \frac{\theta-\theta^{\prime}}{2}\right) .
$$

By unitarity,

$$
S\left(\theta, \theta^{\prime}\right)=\frac{1+\frac{i \kappa}{2}\left(\frac{\cosh \frac{\theta+\theta^{\prime}}{2}}{\sinh \frac{\theta-\theta^{\prime}}{2}}-\operatorname{coth} \frac{\theta-\theta^{\prime}}{2}\right)}{1-\frac{i \kappa}{2}\left(\frac{\cosh \frac{\theta+\theta^{\prime}}{2}}{\sinh \frac{\theta-\theta^{\prime}}{2}}-\operatorname{coth} \frac{\theta-\theta^{\prime}}{2}\right)} .
$$

It is easy to check that in the limit of small momenta this S-matrix reduces to the S-matrix of the LL model.

The S-matrix of the FR model is not Lorentz invariant. This is not surprising because the Virasoro constraints explicitly break the Lorentz symmetry of the originally Lorentz-invariant chiral field. Because of the lack of Lorentz invariance the S-matrix is not a function of $\theta-\theta^{\prime}$, which makes rapidity parametrization not the most convenient one. There is another parametrization of energies and momenta which gives more compact expressions for the S-matrix and the Bethe equations:

$$
\begin{aligned}
& \cosh \theta=\frac{x^{2}+1}{x^{2}-1}, \\
& \sinh \theta=\frac{2 x}{x^{2}-1} .
\end{aligned}
$$


The S-matrix takes an extremely simple form in these variables:

$$
S\left(x, x^{\prime}\right)=\frac{x-x^{\prime}-i \kappa}{x-x^{\prime}+i \kappa} .
$$

We can now write down the Bethe equations:

$$
\exp \left(\frac{2 i \kappa L x_{j}}{x_{j}^{2}-1}\right)=\prod_{k \neq j} \frac{x_{j}-x_{k}+i \kappa}{x_{j}-x_{k}-i \kappa} .
$$

The states with Bethe roots in the interval $-1<x_{j}<1$ carry negative energy. One has to fill all negative-energy levels by constructing a non-trivial vacuum solution of Bethe equations with infinitely many roots. I will not discuss this procedure here, which involves UV regularization and subsequent renormalization. For a lattice-regularized version of the model, the physical vacuum was constructed in [25], which turns out to be a condensate of $2 S$-strings with $S \rightarrow \infty$. The renormalized physical solution describes the quantized principal chiral field as solved in [27] by the fermionization technique.

\section{Conclusions}

A common feature of the calculations presented in this paper is that they are largely insensitive to the full non-linear structure of the sigma-models. The bare S-matrix is completely determined by tree-level quartic vertices. On the other hand, the S-matrix completely determines the exact solution of the model. The deep reason for that is integrability and factorization of the multi-body S-matrix. However, the calculational simplicity and the fact that the bare Bethe equations are valid even in the finite volume are due to the judicious choice of the pseudovacuum. I do not known a general method to find pseudovacuum in an arbitrary integrable field theory, and construction of such a state for the string sigma-model in $A d S_{5} \times S^{5}$ remains an open problem.

Acknowledgements I would like to thank T. Klose for fruitful collaboration on the work presented in this notes, and for numerous illuminating discussions. This was supported in part by the Swedish Research Council under contracts 621-2004-3178 and 621-2003-2742, by the Göran Gustafsson Foundation, and by grant NSh-8065.2006.2 for the support of scientific schools, and by RFBR grant 06-02-17383.

\section{References}

[1] H. Bethe, On The Theory Of Metals. 1. Eigenvalues And Eigenfunctions For The Linear Atomic Chain, Z. Phys. 71, 205 (1931).

[2] L.D. Faddeev, How Algebraic Bethe Ansatz works for integrable model, in Quantum Symmetries, Proceedings of the Les Houches Summer School, Session LXIV, Les Houches, 1 August - 8 September 1995, eds: A. Connes, K. Gawedzki and J. Zinn-Justin [hep-th/9605187].

[3] V.E. Korepin, A.G. Izergin and N.M. Bogolyubov, Quantum Inverse Scattering Method, Correlation Functions and Algebraic Bethe Ansatz, Cambridge Univ. Press, Cambridge 1993.

[4] H. B. Thacker, Exact Integrability In Quantum Field Theory And Statistical Systems, Rev. Mod. Phys. 53 (1981) 253. 
[5] J. M. Maldacena, The large N limit of superconformal field theories and supergravity, Adv. Theor. Math. Phys. 2 (1998) 231 [hep-th/9711200]; S. S. Gubser, I. R. Klebanov and A. M. Polyakov, Gauge theory correlators from non-critical string theory, Phys. Lett. B428 (1998) 105 [hep-th/9802109]; E. Witten, Anti-de Sitter space and holography, Adv. Theor. Math. Phys. 2 (1998) 253 [hep-th/9802150].

[6] R. R. Metsaev and A. A. Tseytlin, Type IIB superstring action in $\mathrm{AdS}_{5} \times S^{5}$ background, Nucl. Phys. B 533, 109 (1998) [hep-th/9805028].

[7] I. Bena, J. Polchinski and R. Roiban, Hidden symmetries of the $\operatorname{AdS}_{5} \times S^{5}$ superstring, Phys. Rev. D 69 (2004) 046002 [hep-th/ 0305116$].$

[8] J. A. Minahan and K. Zarembo, The Bethe-ansatzfor $\mathscr{N}=4$ super Yang-Mills, JHEP 0303, 013 (2003) [hep-th/0212208].

[9] N. Beisert, C. Kristjansen and M. Staudacher, The dilatation operator of $\mathscr{N}=4$ super Yang-Mills theory, Nucl. Phys. B 664, 131 (2003) [hep-th/0303060]; N. Beisert and M. Staudacher, The $\mathscr{N}=4$ SYM integrable super spin chain, Nucl. Phys. B 670, 439 (2003) [hep-th/ 0307042$]$.

[10] A. B. Zamolodchikov and A. B. Zamolodchikov, Factorized S-Matrices In Two Dimensions As The Exact Solutions Of Certain Relativistic Quantum Field Models, Annals Phys. 120 (1979) 253.

[11] M. Staudacher, The factorized S-matrix of CFT/AdS, JHEP 0505, 054 (2005) [hep-th / 0 412188].

[12] N. Beisert, The su(2|2) dynamic S-matrix [hep-th/0511082]; N. Beisert, R. Hernandez and E. Lopez, “A crossing-symmetric phase for $A d S_{5} \times S^{5}$ strings," JHEP 0611 (2006) 070 [arXiv:hep-th/0609044]; N. Beisert, B. Eden and M. Staudacher, Transcendentality and crossing, J. Stat. Mech. 0701 (2007) P021 [arXiv:hep-th/0610251].

[13] N. Beisert and M. Staudacher, Long-range PSU $(2,2 \mid 4)$ Bethe ansaetze for gauge theory and strings, Nucl. Phys. B 727, 1 (2005) [hep-th/ 0504190$].$

[14] J. Ambjorn, R. A. Janik and C. Kristjansen, Wrapping interactions and a new source of corrections to the spin-chain / string duality, Nucl. Phys. B 736, 288 (2006) [arXiv:hep-th/0510171].

[15] S. Schafer-Nameki, M. Zamaklar and K. Zarembo, How accurate is the quantum string Bethe ansatz?, JHEP 0612, 020 (2006) [arXiv:hep-th/0610250]; A. V. Kotikov, L. N. Lipatov, A. Rej, M. Staudacher and V. N. Velizhanin, Dressing and Wrapping, [arXiv:0704.3586 [hep-th] ].

[16] T. Klose and K. Zarembo, Bethe ansatz in stringy sigma models, J. Stat. Mech. 0605, P006 (2006) [hep-th/0603039].

[17] V. E. Korepin, Direct Calculation Of The S Matrix In The Massive Thirring Model, Theor. Math. Phys. 41, 953 (1979) [Teor. Mat. Fiz. 41, 169 (1979)].

[18] H. Bergknoff and H. B. Thacker, Method For Solving The Massive Thirring Model, Phys. Rev. Lett. 42, 135 (1979); Structure And Solution Of The Massive Thirring Model, Phys. Rev. D 19, 3666 (1979).

[19] E.M. Lifshitz and L.P. Pitaevskii, Statistical Physics, Part 2, Pergamon Press, Oxford 1980.

[20] M. Kruczenski, Spin chains and string theory, Phys. Rev. Lett. 93 (2004) 161602 [hep-th/0311203]; M. Kruczenski, A. V. Ryzhov and A. A. Tseytlin, Large spin limit of $\mathrm{AdS}_{5} \times S^{5}$ string theory and low energy expansion of ferromagnetic spin chains, Nucl. Phys. B 692, 3 (2004) [hep-th/0403120].

[21] E.K. Sklyanin, Quantization of the Continuous Heisenberg Ferromagnet, Lett. Math. Phys. 15 (1988) 357. 
[22] L.D. Faddeev and L.A. Takhtajan, Hamiltonian methods in the theory of solitons (Springer-Verlag, 1987).

[23] J. A. Minahan, A. Tirziu and A. A. Tseytlin, 1/J corrections to semiclassical AdS/CFT states from quantum Landau-Lifshitz model, Nucl. Phys. B 735, 127 (2006) [hep-th / 0509071$]$

[24] H. B. Thacker, Bethe's Hypothesis And Feynman Diagrams: Exact Calculation Of A Three Body Scattering Amplitude By Perturbation Theory, Phys. Rev. D 11, 838 (1975); Many Body Scattering Processes In A One-Dimensional Boson System, Phys. Rev. D 14, 3508 (1976).

[25] L. D. Faddeev and N. Y. Reshetikhin, Integrability Of The Principal Chiral Field Model In (1+1)-Dimension, Annals Phys. 167 (1986) 227.

[26] K. Zarembo, Semiclassical Bethe ansatz and AdS/CFT, Comptes Rendus Physique 5, 1081 (2004) [Fortsch. Phys. 53, 647 (2005)] [hep-th/ 0411191 ].

[27] A. M. Polyakov and P. B. Wiegmann, Theory Of Nonabelian Goldstone Bosons In Two Dimensions, Phys. Lett. B 131, 121 (1983). 